\title{
APROVECHAMIENTO DEL ENTORNO NATURAL, UNA ESTRATEGIA DIDÁCTICA PARA MEJORAR EL RENDIMIENTO ACADÉMICO DE JÓVENES EN EL MUNICIPIO DE OTANCHE-BOYACA
}

\section{TAKING ADVANTAGE OF THE NATURAL ENVIRONMENT, A TEACHING STRATEGY TO IMPROVE ACADEMIC PERFORMANCE THE YOUNG PEOPLE OF THE MUNICIPALITY OF OTANCHE- BOYACA}

\author{
Laura Patricia Montes Castellanos'1.Universidad Santo Tomas. Colombia \\ laura.montes@usantoto.edu.co
}

\author{
Luz Ángela Cuellar². Universidad Santo Tomas, Universidad Pedagógica y \\ Tecnológica de Colombia. Colombia \\ luz.cuellar@usantoto.edu.co
}

Este documento es producto de trabajo de investigación de maestría, el documento original se encuentra en el repositorio digital de la universidad Santo Tomás y se presentó en el congreso CIVESA el 29 de febrero de 2020.

\section{RESUMEN}

La investigación presenta la aplicación de una estrategia didáctica que aprovecha el entorno natural presente en la institución educativa San Ignacio de Loyola, con el fin de mejorar la motivación y el rendimiento académico de los estudiantes en el área de ciencias naturales. El trabajo se realizó con estudiantes de grado sexto; para verificar su eficiencia se trabajó con dos grupos, un grupo control, el cual mantuvo la metodología tradicional manejada en la institución y otro experimental el cual fue expuesto a la estrategia. En primer lugar se aplicó una encuesta a los estudiantes para conocer su percepción frente a las prácticas pedagógicas del área, seguida de la aplicación una prueba diagnóstica para conocer el conocimiento previo frente a la temática a trabajar, en este caso ecosistemas, por último se implementó la estrategia con el grupo experimental, está basada en una serie de salidas de campo y guias pedagógicas que contextualizaron temáticas a trabajar. Se determinó que los estudiantes sienten agrado con las practicas que se desarrollan, sin embargo muestran interés por trabajar metodologías innovadoras, en especial aquellas que los involucren directamente en su proceso de aprendizaje, en cuanto a los resultados académicos, el análisis de la prueba diagnóstica arrojo que el nivel de conocimientos de los estudiantes frente al tema era similar, pero luego de la intervención, los resultados favorables se inclinan en un amplio porcentaje hacia el grupo experimental, poniendo en evidencia que estrategias innovadoras y contextualizadas favorecen los procesos académicos en los estudiantes.

\footnotetext{
${ }^{1}$ Laura Patricia Montes Castellanos. Docente de ciencias naturales y educción ambiental en la Institución Educativa San Ignacio de Loyola del municipio de Otanche, Boyacá, Colombia

2 Luz Ángela Cuellar. Docente Investigador Facultad de Ingeniería ambiental, Universidad Santo Tomás, seccional Tunja, Docente Universidad Pedagógica y tecnológica de Colombia, escuela de posgrados.
} 
PALABRAS CLAVE: ciencias naturales, entorno natural, estrategias de enseñanza, metodologías innovadoras, procesos de aprendizaje, motivación, rendimiento académico.

\section{ABSTRACT}

The research presents the application of a didactic strategy that takes advantage of the natural environment present in the San Ignacio de Loyola educational institution, in order to improve the motivation and academic performance of students in the area of natural sciences. The work was carried out with sixth grade students; To verify its efficiency, we worked with two groups, a control group, which maintained the traditional methodology used in the institution, and an experimental group, which was exposed to the strategy. In the first place, a survey was applied to the students to know their perception of the pedagogical practices of the area, followed by the application of a diagnostic test to know the previous knowledge regarding the subject to work, in this case ecosystems, finally it was implemented The strategy with the experimental group is based on a series of field trips and pedagogical guides that contextualize the topics to be worked on. It was determined that the students feel comfortable with the practices that are developed, however they show interest in working with innovative methodologies, especially those that involve them directly in their learning process, in terms of academic results, the analysis of the diagnostic test was bold. that the level of knowledge of the students regarding the subject was similar, but after the intervention, the favorable results incline in a large percentage towards the experimental group, evidencing that innovative and contextualized strategies favor academic processes in students

KEYWORDS: natural sciences; natural environment; teaching strategies; innovative methodologies; learning processes; motivation; academic performance.

\section{Cómo citar el artículo:}

Montes Castellanos, L. P. y Cuellar, L. A. (2020). Aprovechamiento del entorno natural, una estrategia didáctica para mejorar el rendimiento académico de jóvenes en el municipio de Otanche-Boyaca. Revista de Ciencias de la Comunicación e Información, 25(3), 19-39.

doi: http://doi.org/10.35742/rcci.2020.25(3).19-39

\section{INTRODUCCIÓN}

El área de ciencias naturales guarda una estrecha relación con los contextos y el entorno en los que se desenvuelven los individuos, permitiéndoles adquirir información y generar conocimiento a partir de su interacción con el mismo y las experiencias que de esta se desprende. La presente investigación plantea la implementación de una estrategia didáctica basada en la incursión, experimentación y reconocimiento del entorno natural con el que cuenta en la institución educativa, contextualizando las temáticas mediantes guías pedagógicas adaptadas a la realización de talleres y salidas de campo para así favorecer los aprendizajes y por ende el rendimiento académico en el área de ciencias naturales y educación ambiental del grado sexto en la institución educativa san Ignacio de Loyola del 
municipio de Otanche, Boyacá. A partir de esta también se busca mejorar la motivación y el interés por el área.

El porcentaje de bajos resultados académicos en ciencias naturales, es constante en el colegio, según análisis de calificaciones de grado sexto en el área en años anteriores durante los años 2016, 2017 y 2018 más del 50\% los estudiantes se ubicaron en el nivel bajo según la escala de clasificación de la institución aumentando la deserción y perdida del año escolar, lo que genera preocupación en los docentes del área y las directivas de la institución. Ante esta problemática es preciso optar por estrategias didácticas adecuadas y efectivas mitiguen y ayuden a mejorar los resultados antes obtenidos y desencadenen en un satisfactorio resultado académico y actitudinal, en este sentido, los actores activos del proceso educativo deben propender por estrategias didácticas innovadoras que desarrollen destrezas y habilidades para promover en los estudiantes la adquisición de un conocimiento contextualizado que propicie aprendizajes significativos en el área y por ende resultados satisfactorios en la misma.

Al realizar un análisis de la propuesta pedagógica del plan de área de ciencias naturales en la institución, se encuentran elementos básicos de la formación académica como el trabajo autónomo, el desarrollo de habilidades y competencias y claro el logro de aprendizajes significativos. Sin embargo, se evidencia la debilidad que presentan los estudiantes en estos aspectos, evidenciando una mínima apropiación de los conocimientos.

Este trabajo investigativo propone el aprovechamiento de un recurso disponible dentro de la infraestructura educativa, haciendo uso del mismo para diseñar y establecer estrategias didácticas en el área, brindándole a los docentes la posibilidad de direccionar los aprendizajes de los estudiantes dándoles la oportunidad de participar activamente en sus procesos de aprendizaje en pro del mejoramiento académico institucional y el cumplimiento de las exigencias educativas actuales.

\section{OBJETIVOS}

\subsection{Objetivo general}

Verificar el impacto de la implementación de la estrategia didáctica incursión al entorno natural en el mejoramiento del rendimiento académico en los en los estudiantes de grado sexto en ciencias naturales de la institución educativa San Ignacio de Loyola del Municipio de Otanche - Boyacá

\subsection{Objetivos específicos}

1. Utilizar del entorno natural de la institución educativa como herramienta didáctica para mejorar el rendimiento académico de los estudiantes en el área.

2. Diseñar guías didácticas que orienten a los estudiantes a la indagación, exploración y experimentación del medio natural, de acuerdo con los contenidos del plan de área de la institución. 
3. Evaluar la incidencia de la estrategia didáctica en el desempeño académico de los estudiantes en el área y la motivación frente a las estrategias usadas.

\section{METODOLOGÍA}

\subsection{Tipo de investigación}

Para el desarrollo de la investigación se utilizó una metodología mixta, siendo esta la más pertinente para dar respuesta a la pregunta de investigación planteada en el proyecto, en este tipo de investigación se combinan técnicas, métodos, aproximaciones, conceptos o lenguaje cuantitativo y cualitativo dentro de una misma investigación (Johnson y Onwuegbuzie, 2004) con el fin de obtener una visión más completa del fenómeno que se estudia.

Tiene como rasgo sobresaliente el pluralismo metodológico, que según sus defensores, permite derivar en un tipo de investigación de mejor nivel en comparación con una investigación que implica un solo método. De otro lado, el método mixto de investigación puede entenderse como la exploración de las diferencias; un fórum para el diálogo o bien una oportunidad para una mejor comprensión de diferentes vías de ver, conocer y evaluar (Greene y Caracelli, 2003).

Fue seleccionado este método ya que por una parte la investigación cuantitativa da la posibilidad de generalizar, permite evaluar los conocimientos frente al tema y hacer una comparación para medir la eficiencia de la estrategia al hacer la comparación entre los dos grupos que hacen parte de la investigación; de otro lado la investigación cualitativa proporciona profundidad en la información, permitiendo analizar el comportamiento y motivación frente a la estrategia aplicada por el docente, contextualización y análisis general del entorno de aprendizaje en el área

\subsection{Enfoque de la investigación}

El trabajo está orientado bajo la Investigación Acción Educativa (IAE). Esta modalidad, de carácter interpretativo, exploratorio y descriptivo analiza las acciones humanas y las situaciones sociales de una comunidad educativa. Según Sadín (2003), "contribuye a la reflexión sistemática sobre la práctica social y educativa con vistas a la mejora y al cambio tanto personal como social. Unifica procesos considerados a menudo independientes, por ejemplo, la enseñanza, el desarrollo del currículum, la evaluación" (p. 165).

Según Minerva (2006) la IAE: "constituye un proceso de reflexión-acción-cambioreflexión, por y para el mejoramiento de la práctica del docente, mediante la participación activa de este, dirigido a superar los problemas y las necesidades del aula, la escuela y la comunidad, posibilitando el diálogo entre teoría-práctica-teoría" (p. 116).

Para Lewin (1992) con la Investigación Acción se puede lograr simultáneamente, avances teóricos y cambios sociales, mediante unas etapas que consisten en: un diagnóstico de la situación problema, recolección de información, interpretación de la información, diseño de un plan de acción para la solución del problema, puesta en 
marcha y finalmente una evaluación de la estrategia con base en los resultados obtenidos. Así mismo, este tipo de investigación se caracteriza por ser un proceso participativo, colaborativo, que posibilita la autocrítica, permite teorizar la práctica, implica la recopilación, registro y análisis de información.

La Investigación Acción asume la enseñanza como un proceso investigativo, en el cual como se trabajó durante el desarrollo de la investigación intervienen todos los sujetos involucrados en el proceso como coinvestigadores (estudiantes de grado sexto) y el docente como investigador principal, quien dirige y orienta el proceso, permitiendo transformar, mejorar y enriquecer el quehacer docente, reflejado en el éxito académico de sus estudiantes, lo cual se pretende con la realización de este proyecto.

\subsection{Muestra}

Se tomó como muestra los estudiantes de dos cursos de grado sexto de la institución educativa san Ignacio de Loyola, uno grupo control (curso 6-1) con 36 estudiantes y un grupo experimental (curso 6-2), de 36 estudiantes, para un total de 72 niños, los cuales se encuentran entre los 11 y 14 años de edad, provenientes de familias que habitan en su mayoría en la zona rural, que se dedican a prácticas de minería, agricultura y ganadería como medio de subsistencia. Son familias de escasos recursos económicos, donde los adultos acudientes, ya sean padres o abuelos presentas bajos grados de escolaridad.

Al realizar el estudio en dos grupos, uno control y uno experimental, fue posible establecer comparación entre la eficiencia de la estrategia incursión al entorno natural y las practicas pedagógicas tradicionales usadas en el abordaje de los contenidos de las Ciencias Naturales, para la generación de un aprendizaje significativo y por ende el mejoramiento del rendimiento académico.

\subsection{Fases de la investigación}

Para el desarrollo de la investigación se solicitó previamente consentimiento informado a los padres de familia de los estudiantes que participarían en el proyecto, los cuales debían firmar.

El proyecto se desarrolló en tres fases:

- En primer lugar se aplicó una encuesta a los estudiantes de los dos curso de grado sexto validada a juicio de experto (grupo control -grupo experimental) para evidenciar su actitud y motivación en la asignatura y al establecimiento de estrategias innovadoras en el área. Con un total de 5 preguntas se pretendió identificar si los estudiantes presentaban motivación hacia la realización de actividades didácticas como salidas de campo, que les permitieran mejorar los procesos de aprendizaje en las ciencias naturales generando un aprendizaje significativo.

Se aplicó en dos cursos de grado sexto una prueba diagnóstica tipo ICFES (Instituto Colombiano para el Fomento de la Educación Superior) validada a juicio de experto con el fin identificar los conceptos previos de los estudiantes, en la 
temática vista durante el tercer periodo académico según el plan de área de ciencias naturales y educación ambiental de la institución, la cual corresponde a ecosistemas.

La prueba se diseñó con preguntas tomadas del banco de preguntas del ICFES para la prueba de Biología, seleccionando las relacionadas con los temáticos ecosistemas en el grado sexto. Con un total de 25 preguntas, enfocadas al cumplimiento de los derechos básicos de aprendizaje (DBA) y los estándares requeridos propuestos por el ministerio de educación nacional para el grado sexto en el tercer periodo académico. Las preguntas se encuentran agrupadas según cada uno de los estándares de la siguiente manera:

Pregunta 1 a pregunta 11. Estándar 1: caracteriza ecosistemas y analiza el equilibrio dinámico entre sus poblaciones

Pregunta 12 a pregunta 19. Estándar 2: propone explicaciones sobre la diversidad biológica teniendo en cuanta las características climáticas

Pregunta 20 a pregunta 25 estándar 3: establece adaptaciones de algunos seres vivos teniendo en cuanta las características de los ecosistemas en que viven y las relaciones entre ellos

- Iniciando el tercer periodo académico al grupo experimental se aplicaron tres talleres de campo bajo el modelo PTA (programa todos a aprender) validados a juicio de experto enfocados a cada estándar establecido por el ministerio de educación nacional para la temática correspondiente, cada taller contaba con una guía de trabajo dividida en dos partes, un trabajo exclusivamente en campo y una segunda parte en la cual los estudiantes consolidaban los conocimientos que se adquirieron durante la fase de campo mediante una serie de actividades, el grupo control recibió las clases de la misma temática de manera tradicional, en el aula de clase. Los datos obtenidos del grupo experimental durante los talleres de campo fueron registrados utilizando como instrumento el diario de campo.

Diario de campo: Adaptado https://www.mitrabajoessocial.com/guia-paraelaborar-el-diario-de-campo-incluye-plantilla/ Se registró información obtenida a través de la observación en la realización de los talleres de campo realizados con los estudiantes durante el tercer periodo académico

- Terminando el tercer periodo, se aplicó una prueba final (misma prueba diagnóstica) a los dos grupos, con el fin de analizar y comparar los aprendizajes en los dos cursos con respecto al resultado de la prueba diagnóstica, de este modo poder evaluar la eficiencia de la estrategia incursión al entorno natural.

\subsection{Criterios de sistematización y análisis}

La sistematización de la información se hace recurriendo a tablas y figuras utilizadas para organizar los datos de la entrevista y pruebas realizadas, además se recurre a la información registrada en el diario de campo a partir de la observación del desarrollo de las clases, utilizando como estrategia didáctica la incursión al entorno 
natural para evidenciar la motivación y el desarrollo de competencias en los estudiantes durante el desarrollo del proyecto.

La prueba diagnóstica se analizó calculando los aciertos en cada una de las preguntas en los dos grupos (control y experimental), teniendo en cuenta las fortalezas y debilidades en cada uno de los estándares a evaluar para la temática de ecosistemas.

La verificación del aprendizaje de contenidos conceptuales logrados por los estudiantes es producto de la comparación entre la prueba final aplicada a cada uno de los grupos, mediante tablas de frecuencias e histogramas realizados con el programa estadístico SPSS con el fin de determinar la distribución de los datos y su frecuencia, agrupados en los niveles de desempeño según la escala de valoración de la institución y también la media correspondiente a cada grupo, para así dar respuesta concreta a la pregunta de investigación.

\section{RESULTADOS Y DISCUSIÓN}

Los resultados de las encuestas y pruebas realizadas se presentan de la siguiente manera. En primer lugar se muestra el análisis cualitativo, de acuerdo las respuestas dadas por los estudiantes se indaga en su percepción frente al área, sus interés y motivaciones en la misma partiendo de las practicas pedagógicas usadas por los docentes y por otro lado los resultados estadísticos y análisis de la prueba diagnóstica y final en el grupo control y experimental.

\subsection{Percepción y expectativas de los estudiantes frente a al área de ciencias naturales y sus prácticas pedagógicas}

La información obtenida en la encuesta se analizó estableciendo categorías según la percepción y respuestas dadas por los estudiantes en cada pregunta. La prueba se realizó tanto en el grupo control como en el experimental para un total de 72 estudiantes antes de comenzar el tercer periodo académico, es decir antes de la intervención que se hizo con el grupo experimental.

La primera pregunta planteada fue: ¿Considera importante el aprendizaje de ciencias naturales en el colegio para su vida? A la cual, el $100 \%$ los estudiantes contestaron que el aprendizaje de las ciencias naturales es importante debido a factores relacionados con su edad, su contexto y sus sueños. Los estudiantes encuentran relación entre el área de ciencias naturales y el aprendizaje de conocimientos que explican fenómenos de su vida cotidiana y su relación con el entorno. Según Juan Manuel Gutiérrez Vázquez (1984) citado por Fumagalli (2002) Los niños demandan el conocimiento de las ciencias naturales porque viven en un mundo en el que ocurren una enorme cantidad de fenómenos naturales para los que el niño mismo está deseoso de encontrar una explicación. Lo anterior hace necesario que los docentes brinden espacios de aprendizaje con estrategias innovadoras que satisfagan las necesidades de los estudiantes y generen un aprendizaje significativo. 
A la pregunta ¿El desarrollo de las clases de ciencias le genera motivación para aprender los conceptos propios del área? El 68\% de los estudiantes manifestaron sentirse motivados en el aprendizaje de las temáticas del área, un $26 \%$ manifiesta que se sienten poco motivados y un $6 \%$ no tienen motivación por la asignatura. (Gráfico 1)

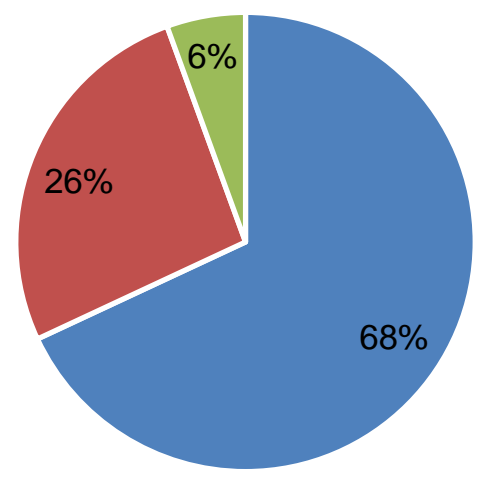

- Mucho

- Poco

- Nada

Gráfico 1. ¿El desarrollo de las clases de ciencias le genera motivación para aprender los conceptos propios del área?

Fuente: elaboración propia.

La motivación no es un factor que se pueda atribuir únicamente a características personales del estudiante, su presencia o ausencia se ve influenciada también por las relaciones entre el alumnado y sus profesores y los procesos pedagógicos que los docentes realizan. De acuerdo con Ausubel, la clave de la motivación está en el interés que se cree por un nuevo aprendizaje. Los resultados revelan que aunque la mayoría siente motivación un amplio grupo está del lado opuesto, lo que suscita una dificultad en la generación de un aprendizaje significativo en las temáticas del área. Según Pomar (2001) uno de los aspectos que más atrae a los estudiantes y aumentan su motivación es la posibilidad de descubrir y comprender por sí mismo aspectos relacionados con su cotidianidad, por lo anterior, es función del docente privilegiar actividades que involucren los recursos del medio, aquellas que le permiten aproximar al niño a los elementos y características del entorno para dotarlos de experiencias que faciliten su aprendizaje, aumentando su interés y motivación en el área.

Por lo anterior se planteó a los estudiantes la pregunta ¿Cree que las actividades realizadas en las clases de ciencias naturales son suficientes para lograr un aprendizaje significativo? A la cual el $90 \%$ de los mismos manifestaron que las actividades realizadas en el área les ayudan a lograr aprendizajes significativos en el área (Gráfico 2) 


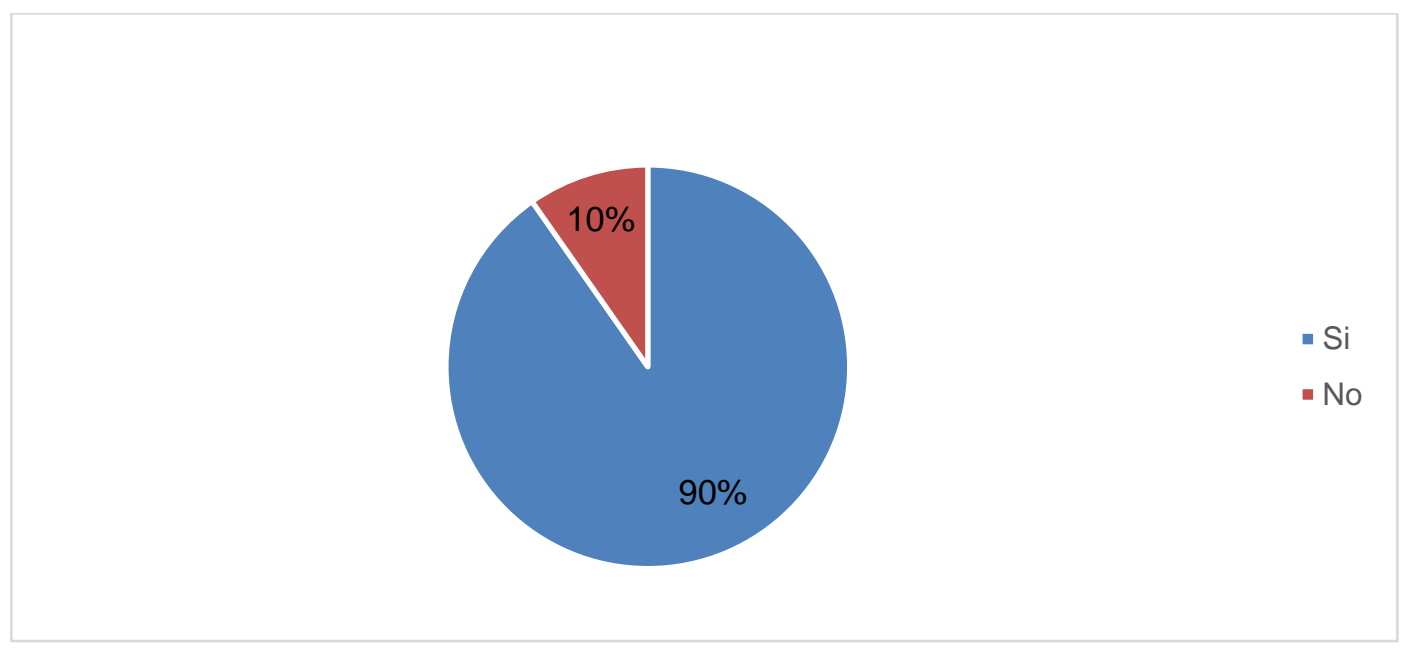

Gráfico 2. ¿Cree que las actividades realizadas en las clases de ciencias naturales son suficientes para lograr un aprendizaje significativo?

Fuente: elaboración propia.

Dentro de las respuestas dadas por los estudiantes, estos manifiestan que la explicación del docente es la adecuada para comprender las temáticas, además demuestran interés por las mismas por lo cual son fáciles de entender. Sin embargo al momento de ser evaluadas los resultados muestran un fenómeno contario poniendo en evidencia que un amplio porcentaje de los estudiantes no comprendió el tema visto, lo anterior refleja, que la metodología empleada por los docentes en el proceso de enseñanza aprendizaje, sigue siendo memorística y momentánea, ya que no crea ningún impacto ni proceso en el estudiante que le permita relacionarla y comprenderla para lograr interiorizarla. Bruner, atribuye una gran importancia a la actividad directa de los estudiantes sobre la realidad, para así lograr una contextualización del aprendizaje que le permita asociarlo y recordarlo con facilidad, en relación con lo planteado por Bruner, pedagogos como Ausubel y Novak sostienen que el aprendizaje debe ser significativo y no memorístico, para ello los nuevos conocimientos deben relacionarse con los saberes previos del estudiante poniendo en evidencia la necesidad de generar estrategias innovadoras y contextualizadas que favorezcan el aprendizaje significativo.

Teniendo en cuenta lo anterior se preguntó a los estudiantes si les gustaría que se implementaran otro tipo de estrategias en el área, diferentes a las usadas por su docente, a la cual el $68 \%$ contesto de forma afirmativa (Gráfico 3) deseando que se implementen nuevas estrategias en el área, según las respuestas obtenidas en la entrevista los estudiantes hacen referencia a actividades como Videos, salidas pedagógicas, laboratorios, entre otras. 


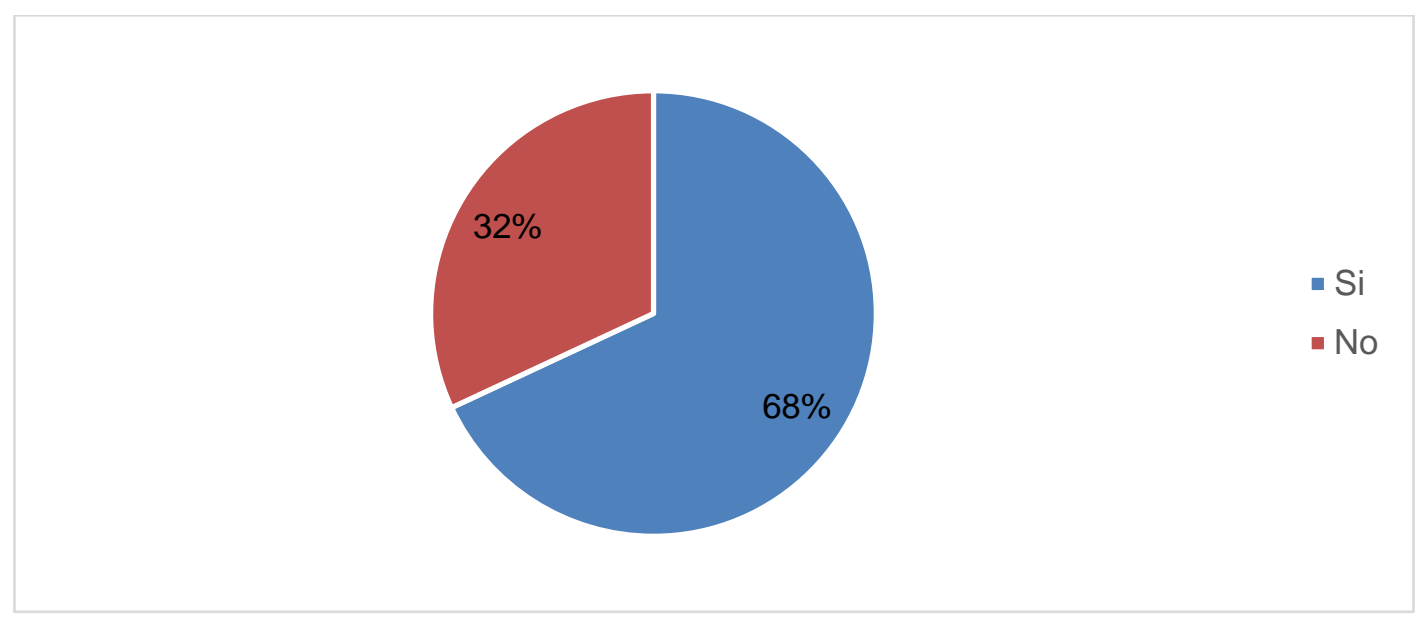

Gráfico 3. ¿Le gustaría que se implementaran otro tipo de estrategias en el área, diferentes a las usadas por su docente?

Fuente: elaboración propia.

Esto pone en evidencia la necesidad de reemplazar los métodos tradicionales que la mayoría de veces resultan tediosos y poco motivantes, por herramientas más didácticas, que permitan tener más dinamismo en las clases e interacción entre los actores de la misma, Vigotzky sostiene que es importante que el docente cree condiciones necesarias que brinden al estudiante experiencias imprescindibles para la formación de conceptos. Para esto, los materiales didácticos se convierten en mediadores dirigidos al logro de esta función, en el momento de dar la clase, Ausubel afirma que los medios y la manera en cómo se trasmite el mensaje juega un papel fundamental en el aprendizaje del individuo. Por lo anterior los docentes deben tener disposición para establecer estrategias más didácticas que favorezcan los procesos de enseñanza - aprendizaje, generando un impacto positivo y de apropiación del conocimiento, y esto a su vez se refleje en los resultados académicos de los estudiantes.

Como se ha mencionado anteriormente, es sabido por los docentes y sustentado por varias investigaciones que las estrategias de que se utilizan en clase les facilitan a los estudiantes la aprehensión del conocimiento, permitiéndoles lograr aprendizajes significativos y logros exitosos, teniendo en cuenta el papel activo del estudiante en su proceso formativo con el fin de conocer su percepción, para esto se planteó la pregunta ¿Las estrategias pedagógicas y didácticas usadas por el docente de ciencias naturales influyen en su rendimiento académico en el área? mostrando que el $74 \%$ de los estudiantes consideran que las estrategias que se usan en el área (laboratorios, videos, talleres grupales) si influyen en su rendimiento académico, (Gráfico 4) manifestando que gracias a estás han logrado entender mejor los conceptos relacionados al área o han visto una mejora en su rendimiento desde su puesta en práctica. 


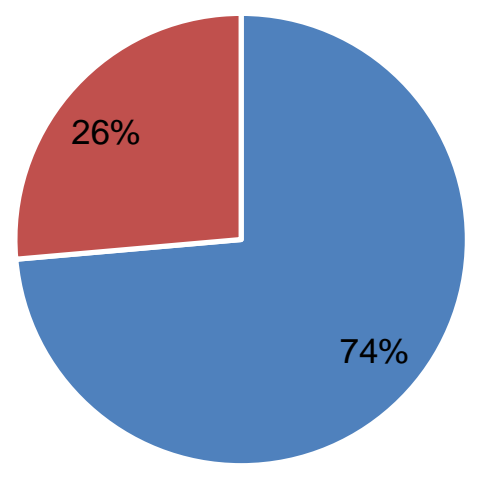

Gráfico 4. ¿Las estrategias pedagógicas y didácticas usadas por el docente de ciencias naturales influyen en su rendimiento académico en el área?

Fuente: elaboración propia.

De acuerdo a esto, Porlán (2000) sugiere que los procesos de enseñanza deben influir en el aprendizaje de los niños para facilitar la apropiación de conocimientos, de formas de pensar y actuar que no pueden aprender de forma espontánea. Por eso, las estrategias usadas el ejercicio de la práctica pedagógica deben estar encaminadas al cumplimiento de estas metas.

\subsection{Análisis prueba diagnóstica y prueba final}

Para el análisis de esta prueba se realizaron graficas de cada una de las preguntas en las dos pruebas para cada grupo a partir de estos resultados, se hizo una comparación de los tres estándares a cumplir según el MEN en el tercer periodo académico para grado sexto, antes y después de la implementación de la estrategia.

\subsubsection{Análisis por estándares}

La prueba diagnóstica aplicada a cada uno de los grupos tenía como objetivo determinar el nivel de conocimientos de los estudiantes respecto a un tema específico, el cual comprende tres estándares para grado sexto. Teniendo en cuenta la procedencia de los estudiantes que en su mayoría es rural se espera tener resultados similares en los dos grupos, además poner en evidencia los conceptos previos de cada estudiante respecto al tema ya haya sido adquirido en años escolares anteriores o producto de su propia experiencia, por otro lado la prueba final se realiza con el fin de establecer una comparación en cuanto a los resultados entre el grupo experimental, en el cual se aplicó la estrategia incursión al entorno natural, y el grupo control con el cual se trabajó de forma tradicional en el aula de clase transcurrido el tercer periodo académico en el cual se desarrolla la temática correspondiente a ecosistemas, de lo anterior se obtuvo los siguientes resultados. 


\section{Estándar 1: Caracteriza ecosistemas y analiza el equilibrio dinámico entre sus poblaciones}

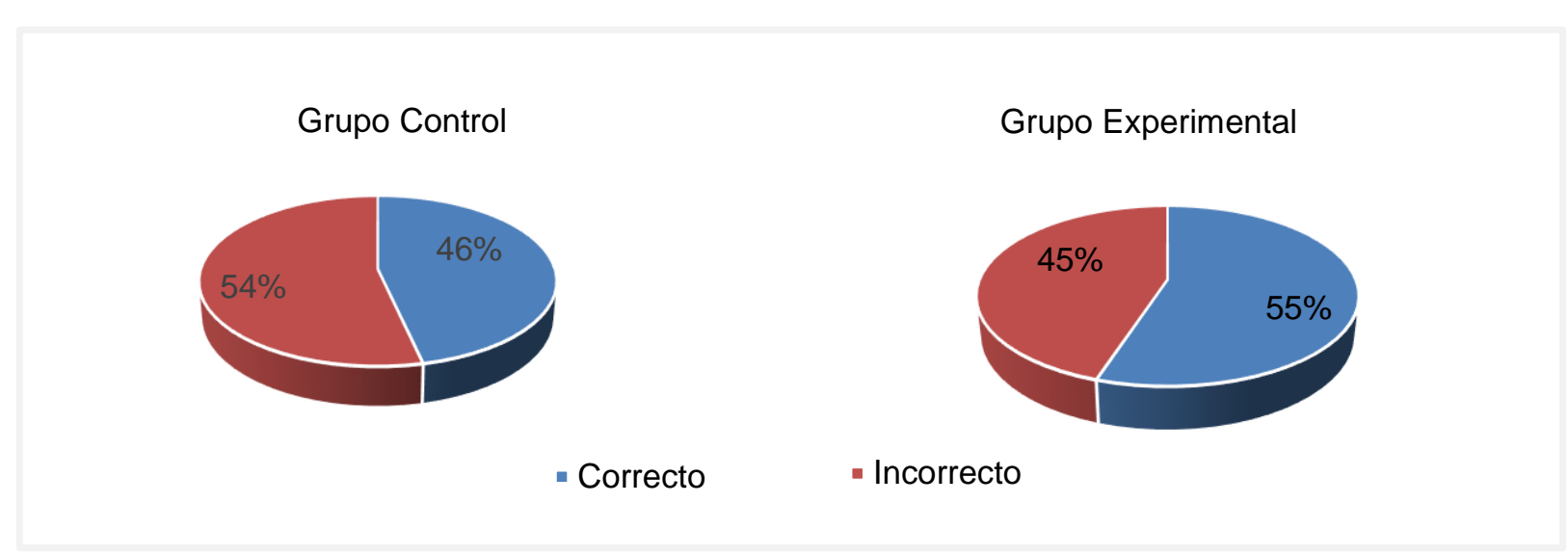

Gráfico 5. Resultados prueba diagnóstica estándar 1

Fuente: elaboración propia.

Los resultados obtenidos en la prueba diagnóstica muestran un mejor desempeño en las preguntas enfocadas al cumplimiento del estándar 1 para el grupo experimental, como se muestra en el Gráfico 5.

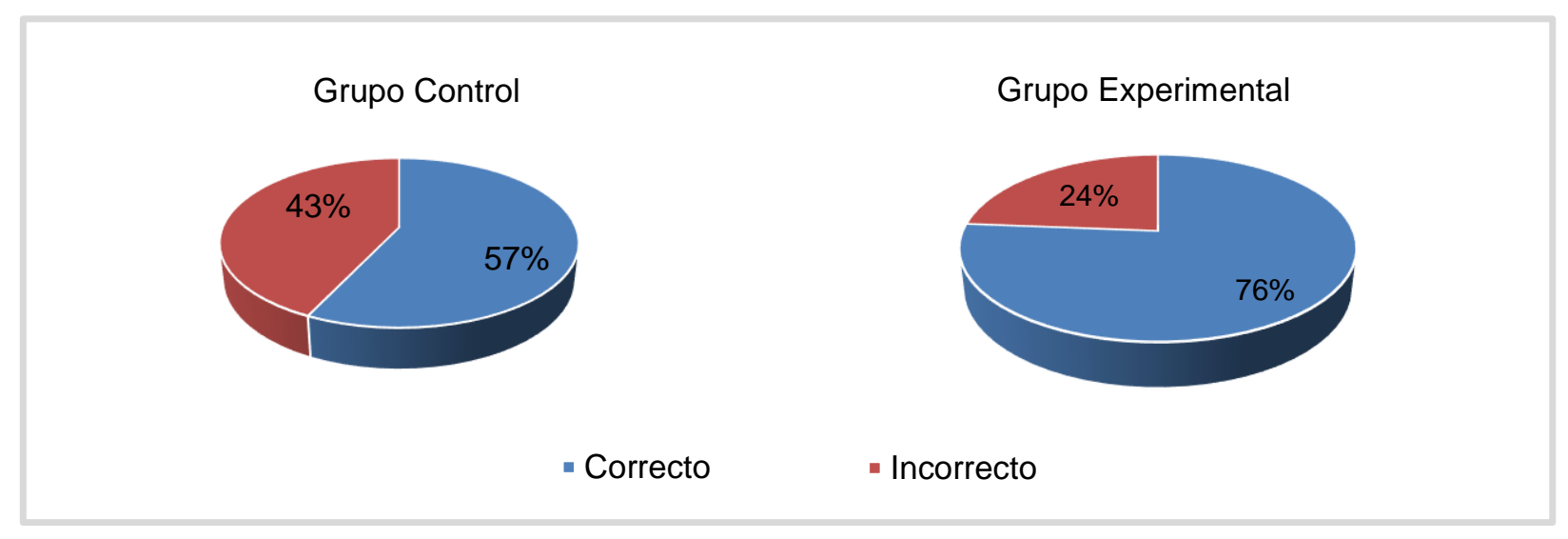

Gráfico 6. Resultados prueba final estándar 1

Fuente: elaboración propia.

En el Gráfico 6, se observa que el grupo experimental obtuvo un mejor desempeño en las preguntas enfocadas al estándar 1, con respecto al grupo control, aunque los dos grupos presentaron un mejor resultado en comparación con a prueba diagnóstica, este fue mayor en el grupo experimental 


\section{Estándar 2: Propone explicaciones sobre la diversidad biológica teniendo en cuenta las características climáticas}

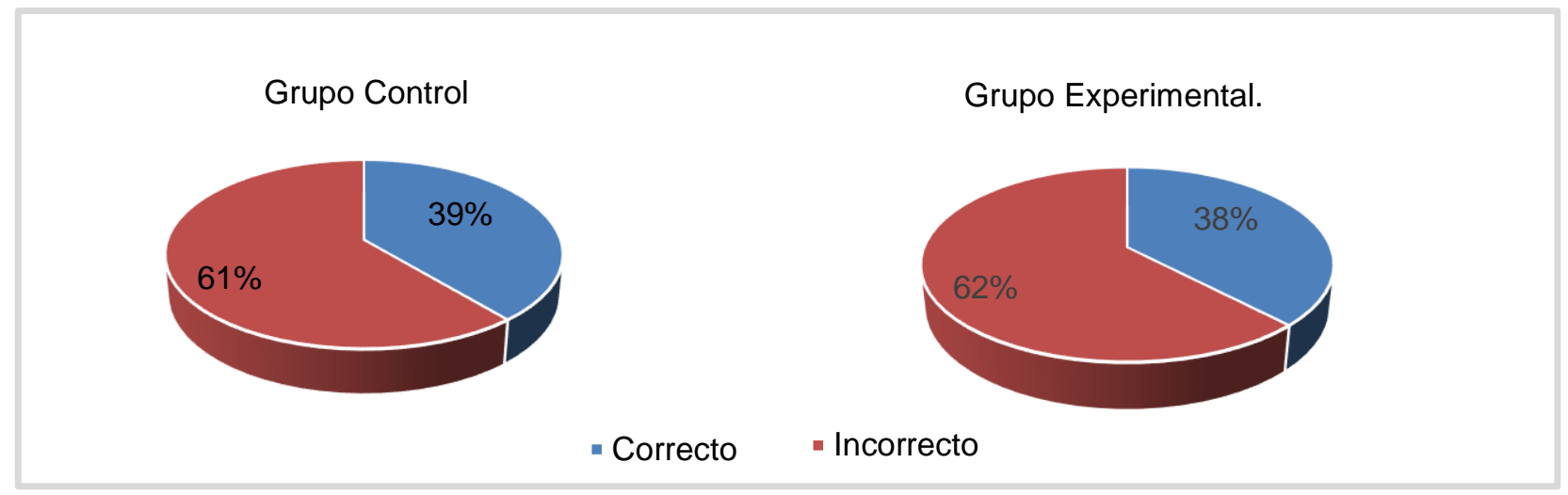

Gráfico 7. Resultados prueba diagnóstica estándar 2

Fuente: elaboración propia.

Los resultados obtenidos en la prueba diagnóstica muestran un mejor desempeño en las preguntas enfocadas al cumplimiento del estándar 2 para el grupo control, con una diferencia no muy amplia del 1\% (Gráfico 7)

Grupo Control.

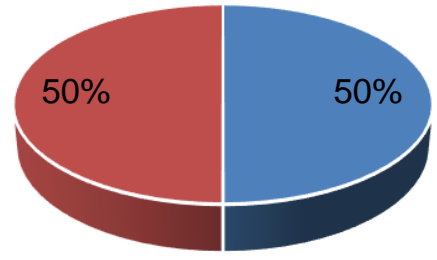

Grupo Experimental.

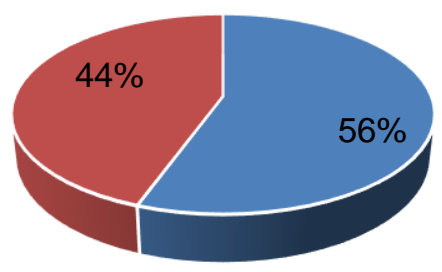

- Correcto - Incorrecto

Gráfico 8. Resultados prueba final estándar 2

Fuente: elaboración propia.

Se observa en el Gráfico 8 que el grupo experimental obtuvo un mejor desempeño en las preguntas enfocadas al estándar 2, con respecto al grupo control, aumentando la diferencia en porcentaje en comparación con la prueba diagnóstica lo que evidencia un mayor acercamiento de los estudiantes del grupo experimental al cumplimiento del estándar. 


\section{Estándar 3: Establece adaptaciones de algunos seres vivos teniendo en cuenta las características de los ecosistemas en que viven y las relaciones entre ellos.}

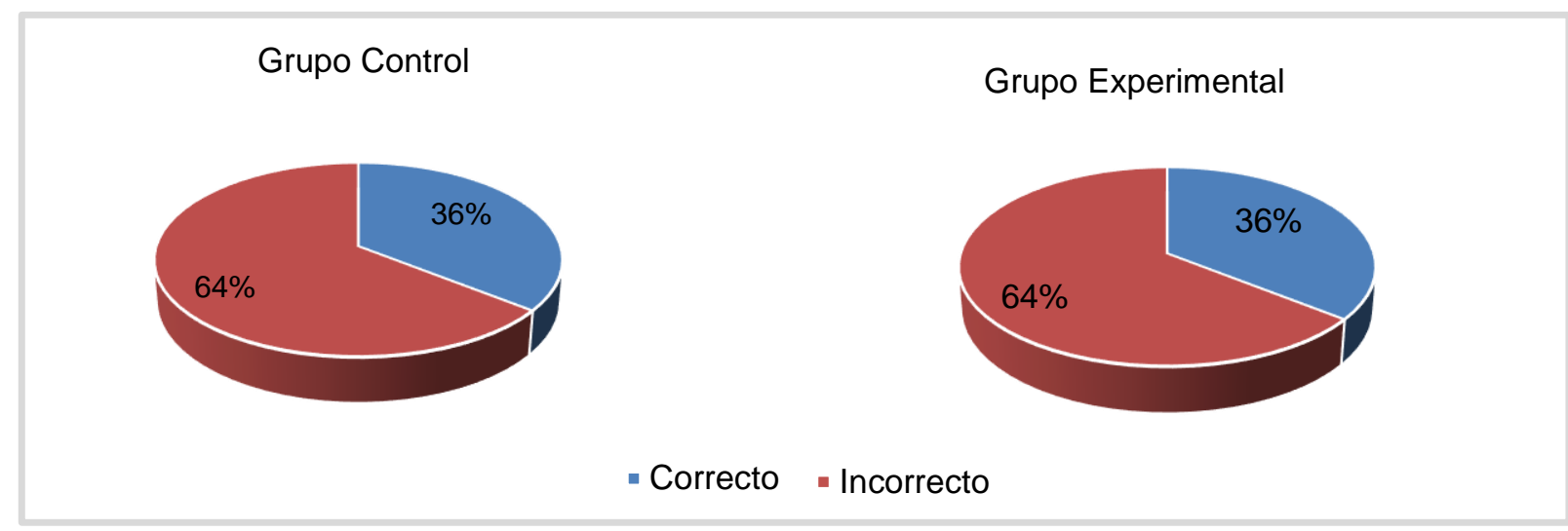

Gráfico 9. Resultados prueba diagnóstica estándar 3

Fuente: elaboración propia.

En los resultados obtenidos en la prueba diagnóstica se observa que los dos grupos no presentan diferencia en porcentaje, en relación al estándar 3. (Gráfico 9)

Grupo Control

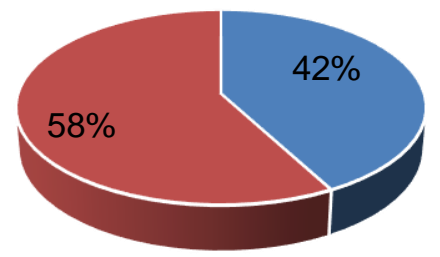

Grupo Experimental

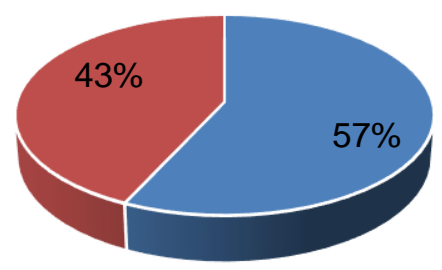

- Correcto - Incorrecto

Gráfico 10. Resultados prueba final estándar 3

Fuente: elaboración propia.

Se observa en el Gráfico 10, que el grupo experimental obtuvo un mejor desempeño en las preguntas enfocadas al estándar 3, con respecto al grupo control, aunque los dos grupos mejoraron sus resultados en comparación con la prueba diagnóstica, el grupo experimental obtuvo un porcentaje mayor.

Los resultados anteriores dan cuenta, que los estudiantes tienen un conocimiento previo en relación al tema en estudio específico, reflejado en los resultados de la prueba diagnóstica, ya sea como parte de su proceso escolar o resultado de sus propias vivencias, al provenir los estudiantes de zonas rurales, su contacto con la medio ambiente es cotidiano, lo que les permite reconocer fenómenos y situaciones que ocurren en la naturaleza. Según lo anterior, los estudiantes a lo largo de su desarrollo han forjado aprendizajes, estos para una mejor comprensión y análisis deben ser estructurados en la escuela, Según, Chevallard (1985), "la ciencia escolar 
no es la ciencia de los científicos, pero existe un proceso de transposición didáctica del conocimiento científico al ser transmitido en el contexto escolar de enseñanza" (p.23). Lo anterior señala el papel fundamental del docente al proporcionar a los estudiantes herramientas, métodos o estrategias que le permitan relacionar y transformar sus aprendizajes aproximándolos al conocimiento científico, para así lograr una mayor aprehensión de los mismos contribuyendo a ampliar y enriquecer sus saberes.

Los resultados de la prueba final, la cual se desarrolló después de la implementación de la estrategia incursión al entorno natural en el grupo experimental, muestran un mejor desempeño de los estudiantes en comparación con el grupo control, el cual no presentó ninguna intervención, poniendo en evidencia que al vivenciar experiencias relacionadas con un aprendizaje, en este caso al estar en contacto con la naturaleza los estudiantes relacionan mejor sus conceptos previos, adquieren mayor seguridad a la hora de expresarlos, clarifican sus ideas y construyen un conocimiento que va más allá de lo memorístico permitiéndoles la aprehensión del mismo y que a su vez este persista, viéndose esto reflejado en su rendimiento académico

\subsubsection{Análisis prueba de normalidad}

Se muestran a continuación las tablas y graficas arrojadas por el programa SPSS durante la sistematización de datos. Se debe tener en cuenta que la escala de valoración de la institución educativa va de 1 a 5, distribuyendo los desempeños como se muestra en la siguiente tabla:

Tabla 1. Valores cuantitativos de nivel de desempeño

\begin{tabular}{|c|c|}
\hline Nivel de desempeño & Rango \\
\hline SUPERIOR & $4,5-5.0$ \\
\hline ALTO & $4,0-4,4$ \\
\hline BÁSICO & $3,0-3,9$ \\
\hline BAJO & $1-2,9$ \\
\hline
\end{tabular}

Fuente: Institución educativa San Ignacio de Loyola.

Para la realización de los gráficos los datos se agruparon con un rango de una unidad de diferencia, teniendo como dato mínimo 1 y dato máximo 5 Resultados prueba Final 


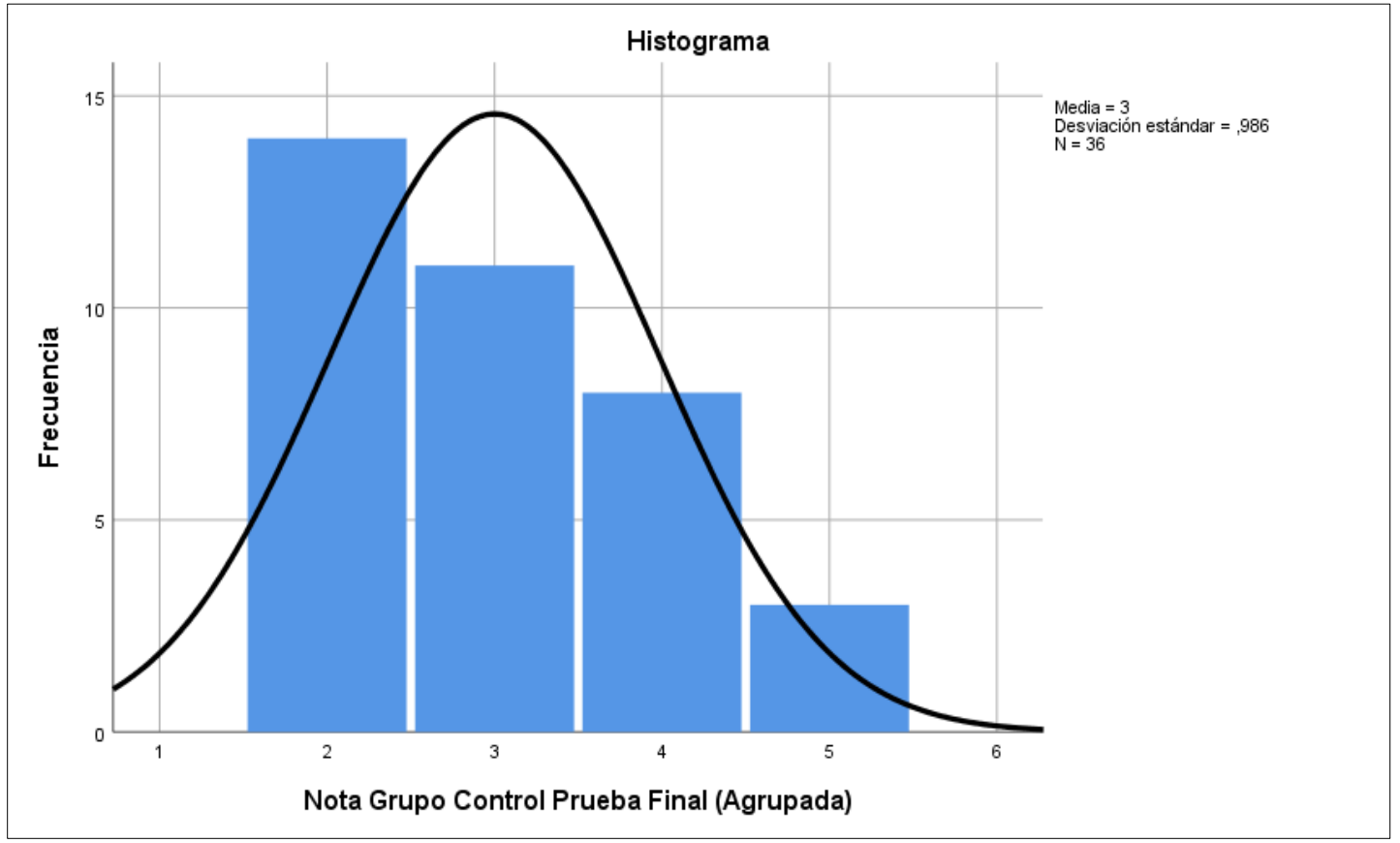

Gráfico 11. Prueba de normalidad grupo control

Fuente: elaboración propia.

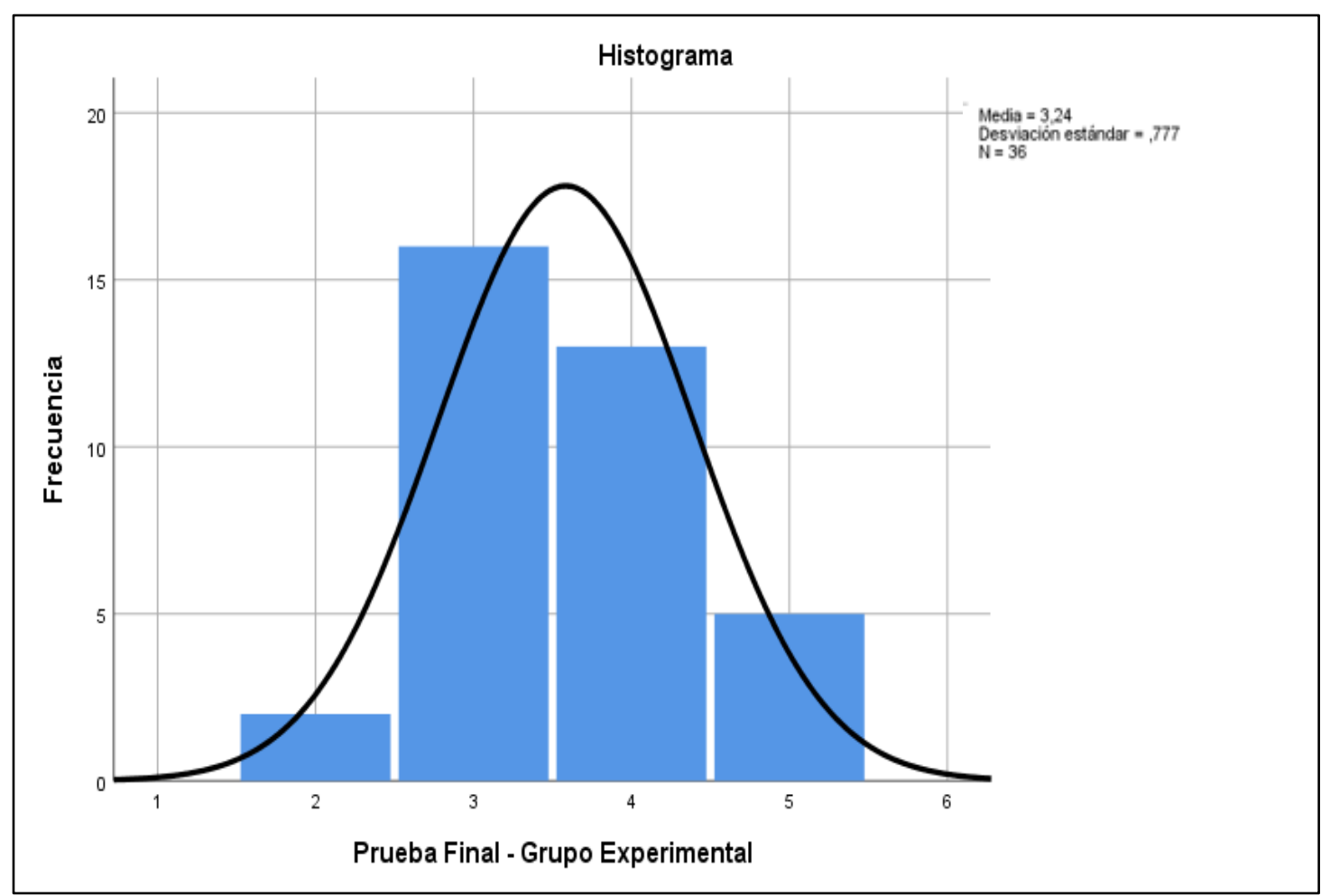

Gráfico 12. Prueba de normalidad grupo experimental

Fuente: elaboración propia.

En los gráficos 11 - 12 se observan los histogramas de la prueba final para el grupo control y experimental con una curva normal ajustada superpuesta, el histograma del grupo control (gráfico 11) muestra una distribución con una cola hacia la derecha, es 
decir, que la mayoría de los estudiantes en el grupo control obtuvieron notas bajas en su mayoria entre 1.5 y 2.9, seguido por promedios de 3.0 a 3.5 despues de ver la temática ecosistemas, en comparacion con la prueba diganostica ningun estudiante obtuvo la calificacion minima, aunque la mayoria se encuantran en el nivel bajo y basico, el 8,3\% de los estudiantes lograron alcanzar un nivel alto con notas entre 4.1 y 4.4, nivel que no se presento en este grupo en la prueba dignostica, no hubo resultados de desempeños superiores.

En el histograma arrojaodo para el grupo experimental (Gráfico 12) se observa una dismunicion significativa de estudiantes con notas bajas, inferiores a 2,9, en comparación con la prueba diagnostica, en este grupo la mayoria de los estudiantes de encuntran en los niveles de desempeño basico y alto, con notas entre 3.0 y 4.4, se registraron 4 resultados para el nivel superior.

Los resultados anteriores dan cuenta de la mejora en el desempeño academico de los estudiantes expuestos a la implementación de la estrategia incursion al entono natural, evidenciando un aumneto en los desempeños de los estudiantes con respecto al grupo control y los resultados obtenido en año anterior, en la misms tematica, durante el mismo periodo.

\subsection{Análisis talleres de campo}

Para el análisis de resultados obtenidos en la realización a las salidas de campo, se emplean siglas de acuerdo al número de salidas realizadas como se muestra en la siguiente tabla

Tabla 2. Abreviaciones diario de campo

\begin{tabular}{|l|l|}
\hline \multicolumn{2}{|c|}{ Tabla de siglas } \\
\hline Abreviación & Descripción \\
\hline DC. 1 & Diario de Campo 1 \\
\hline DC. 2 & Diario de Campo 2 \\
\hline DC. 3 & Diario de Campo 3 \\
\hline
\end{tabular}

Fuente: elaboración propia.

Se logró determinar que mediante las salidas de campo los estudiantes aumentan su agrado y motivación por el área de ciencias naturales, la curiosidad y cuestionamiento continuo se ve favorecido al estar en contacto directo con los fenómenos a estudiar (DC. 1, DC.2, DC. 3).

Durante la realización de cada uno de los talleres de campo, mediante observación se puedo determinar que los estudiantes desarrollan la guía de campo exigiendo un acompañamiento docente continuo siguiendo las orientaciones de este, a medida que trascurre el trabajo en campo los estudiantes asumen el reto de querer realizar el actividad por sí solos, aunque acuden constantemente al docente para tener su aprobación (DC.1) siguiendo las actividades de la guía se evidencia el favorecimiento del aprendizaje colaborativo, algunos estudiantes asumen roles de líderes, ayudando y aconsejando a sus compañeros en la realización de las actividades. 
Lo estudiantes manifiestan su agrado con la actividad realizada, argumentando el comprender algunos procesos vistos con anterioridad y el aprendizaje de cosas nuevas, también demuestran curiosidad y asombro por conocimiento de temas referentes a las ciencias naturales (DC. 2) sienten emoción y satisfacción al plantear sus propias hipótesis y deducciones que son aprobadas por sus compañeros o el docente, evidenciando su motivación y gusto por la temática que se está trabajando al poder relacionarla con lo observan en su entorno.

Durante la tercera salida, se observa un desprendimiento estudiante- docente aunque el docente interactúe con ellos mostrando disposición para atender las inquietudes y necesidades, los estudiantes prefieren realizar las actividades ellos solos o apoyarse en sus mismos compañeros (DC. 3).

El docente indaga a través de preguntas para identificar la evolución en el aprendizaje de los estudiantes, apoyando esta indagación en diversas actividades que ponen en evidencia la apropiación del conocimiento (DC. 1, DC.2, DC. 3). Según Fumagalli (2002), la ciencia escolar está constituida por contenidos conceptuales, procedimentales y actitudinales, lo cual da lugar a muchos espacios en que los estudiantes puedan poner en evidencia los aprendizajes logrados y por tanto dar pautas al docente para guiar el proceso hacia la construcción del conocimiento. Esto se vio favorecido al realizar las salidas de campo, pues brinda al docente un nuevo espacio y herramientas diferentes a las tradicionales usadas en el aula de clase para constatar los aprendizajes de los estudiantes, sumado a esto durante las salidas de campo se contó con la participación activa del total de estudiantes (DC. 1, DC.2, DC. 3). Donde se observó una reflexión continua y cuestionamiento sobre algunos fenómenos de los entornos visitados.

La puesta en práctica de estrategias que involucren la participación activa de los estudiantes en su proceso de aprendizaje, evidencia una mejor disposición de los mismos en cuanto a interés, motivación y la apropiación de los conocimientos referentes a la temática vista al poder relacionarla con su entorno, pues, como sostiene Fumagalli 2002, es necesario acudir a múltiples estrategias de enseñanza que faciliten la apropiación activa de contenidos conceptuales, procedimentales y actitudinales, logrando así enriquecer el conocimiento espontáneo de los estudiantes.

\section{CONCLUSIONES}

Los resultados y análisis obtenidos mediante el proceso de investigación permiten concluir que:

La implementación de la estrategia incursión al entorno natural permitió a los estudiantes reforzar sus saberes previos y adquirir nuevos conocimientos de una manera contextualizada, mejorando su motivación frente a la asignatura y el rendimiento académico en la misma facilitando el desarrollo de habilidades investigativas y favoreciendo actitudes positivas como la responsabilidad, el compañerismo y el compromiso. 
Se evidencio la necesidad de modificar las prácticas de enseñanza tradicionales y fomentar metodologías recurriendo a los recursos disponibles del medio que respondan a las necesidades formativas actuales en pro del mejoramiento académico de los estudiantes y por ende del mejoramiento institucional.

El impacto de la estrategia implementada desde el enfoque del aprendizaje significativo para la enseñanza de las ciencias naturales, no solo aporto en la mejora de los resultados académicos, fomento habilidades y destrezas en las estudiantes propias del quehacer investigativo.

Se pudo determinar el gran impacto que genera en los estudiantes el material didáctico guía usado por los docentes, ya que este juega un papel fundamental en el aprendizaje, pues facilita el acceso de los niños al conocimiento estimula su creatividad y a su vez enriquece la práctica educativa.

Estrategias que involucren como sujeto activo del aprendizaje al estudiante favorecen su nivel de responsabilidad con el mismo, con sus compañeros y con el área, dándole autonomía y seguridad en su proceso de aprendizaje, permitiéndole alcanzar las metas propuestas en el área de manera acertada.

Terminada la investigación se hace evidente la diferencia entre los resultados de los dos grupos involucrados, favoreciendo la implementación de estrategias innovadoras y contextualizadas que beneficien los procesos de aprendizaje de los estudiantes. Esta propuesta genera una salida a aprendizajes basados en transmisión y recepción de manera mecánica, que no generan ningún impacto en los estudiantes, ocasionado poca motivación y mal rendimiento académico.

La estrategia que se plantea en esta investigación es transferible y replicable en otras instituciones educativas, buscando mejorar los procesos de enseñanza aprendizaje contextualizando temáticas del área involucrando a los estudiantes en sus procesos académicos y a su vez despertando la motivación e interés por su formación integral.

\section{REFERENCIAS BIBLIOGRAFÍAS}

Ausubel, David. (1976). Descripción: Psicología educativa. Un punto de vista cognitivo. México: Editorial Trillar.

Ausubel, D. (1978). Citado por Ontoria y cols. En: Mapas conceptuales. Séptima edición. Madrid: Editorial Narcea; 1997: 18.

Bruner, J.S., Goddnow, J. y Austin, G.A. (1978). El proceso mental en el aprendizaje. Madrid: Narcea.

Chevallard, Y. (1985). La transposition didactique; du savoir savant au savoir enseigné. Paris : La Pensée Sauvage. 
Colombia Aprende - $\quad$ Guía del estudiante. http://aprende.colombiaaprende.edu.co/sites/default/files/naspublic/plan choco/Cl ENCIAS 7 BIM2 SEM2 EST.pdf

Fumagalli, L. (2002). La Enseñanza de las Ciencias Naturales en el Nivel Primario de Educación Formal. Argumentos a su Favor. En H. Weissmann, Didactica de las Ciencias Naturales Aportes y Reflexiones (pág. 290). Argentina: Paidos.

Greene, J., \& Caracelli, V. (2003). Making paradigm sense of mixed methods practice. En: A.Tashakkori \& Ch. Teddlie (Ed.), Handbook of mixed methods in social \& behavioral research (pp. 91-110). London: SAGE Publications.

Johnson, R. B., \& Onwuegbuzie, A. J. (2004). Mixed methods research: A research paradigm whose time has come. Educational Research, 33(7), 14-26. Doi: https://doi.org/10.3102/0013189X033007014

Lewin. K. (1992). La investigación-acción y los problemas de las minorías. En La Investigación-acción participativa: inicios y desarrollos, (pp. 13-25). Madrid: Editorial Popular. (Obra original publicada en 1946)

Minerva, F. (2006). El proceso de investigación científica. Zulia, Venezuela: Universidad del Zulia.

Ministerio de Educación Nacional (2006). Estándares Básicos de Competencias en Lenguaje, Matemáticas, Ciencias y Ciudadanas: Guía sobre lo que los estudiantes deben saber y saber hacer con lo que aprenden. Colómbia: Ministerio de Educación Nacional. Recuperado de https://www.mineducacion.gov.co/1621/articles-340021 recurso 1.pdf

Ministerio de Educacion Nacional (2015). Investigación de los Saberes Pedagógicos. Colombia: Ministerio de Educación Nacional. Recuperado de http://www.mineducacion.gov.co/1621/articles-345504 anexo 13.pdf

Porlán, R. (2000). Hacia un Modelo de Enseñanza - Aprendizaje de las Ciencias por investigación. En M. Kaufman, \& L. Fumagalli, Enseñanza de las Ciencias Naturales - Reflexiones y Propuestas Didácticas (pág. 270). Argentina: Paidós Educador.

Pomar, C.M. (2001). La motivación de los superdotados en el contexto escolar. Santiago de Compostela: Universidad de Santiago de Compostela.

Sandín, M. (2003). Investigación cualitativa en educación: fundamentos y tradiciones. Madrid: McGraw-Hill.

Secretaría de educación de Boyacá - Institución Educativa San Ignacio de Loyola. (2015). Sistema de información SIMAT. Otanche.

Vygotsky L. (1979). El desarrollo de los procesos psicológicos superiores. Barcelona: Grijalbo. 


\section{AUTORAS}

Laura Patricia Montes Castellanos

Universidad Santo Tomas. Colombia

laura.montes@usantoto.edu.co

\section{Luz Ángela Cuellar}

Universidad Santo Tomas, Universidad Pedagógica y Tecnológica de Colombia.

Colombia

luz.cuellar@usantoto.edu.co 